\title{
Visual perception of surface curvature: Psychophysics of curvature detection induced by motion parallax
}

\author{
VALÉRIE CORNILLEAU-PÉRÈS \\ Essilor, Paris, France \\ and \\ JACQUES DROULEZ \\ Laboratoire de Physiologie Neurosensorielle, C.N.R.S., Paris, France
}

\begin{abstract}
The continuous approach to optic-flow processing shows that the curvature of a moving surface is related to a second spatial derivative of the velocity field, the spin variation (Droulez \& Cornilleau-Pérès, 1989). With this approach as a theoretical framework, visual sensitivity to the curvature of a cylinder in motion was measured using a task of discrimination between cylindrical and planar patches. The results confirm the predictions suggested by the theory: (1) Sensitivity to curvature was always greater when the cylinder axis and the frontal translation were parallel than when they were orthogonal. The ratio of curvature detection thresholds in the two cases was between 1.3 and 2.5; the value predicted from the spin variation theory is about 2. (2) Sensitivity to curvature increased strongly with the velocity of the motion but was only weakly affected by its amplitude and the duration of viewing for the range of values used in our experiments.
\end{abstract}

Moving and acting in the physical world requires the observer to continually form a coherent representation of the three-dimensional (3-D) environment. This is achieved by the use of various sources of information, among which visual afferences are predominant. The study of the visual perception of the 3-D characteristics of objects is therefore of fundamental importance. This paper focuses in particular on the perception of surface curvature, a choice which was motivated by a set of observations concerning wearers of eyeglasses.

Lenses designed for optical correction or for experimental purposes, such as those used by Droulez and Cornilleau (1986), induce metrical distortions of the visual field and yield erroneous perceptions of surface curvature, particularly as the viewer moves during self-motion. The viewer is then greatly bothered by the apparent curvature of usually flat surfaces, such as the ground or walls. This phenomenon raises the question of human visual sensitivity to surface curvature (e.g., second-order variations of a smooth depth map).

Depth perception is ensured by various visual cues, of which motion parallax and binocular disparity are classically recognized as the most precise and reliable. As a preliminary study of the perception of surface curvature, the approach described here uses motion parallax as the only cue to depth.

This work was supported by Grant ATP/Communication from the Centre National de la Recherche Scientifique. We thank M. Ehrette for constructing the experimental apparatus. Correspondence may be sent to Valérie Cornilleau-Pérès, Laboratoire de Physiologie Neurosensorielle, 15 Rue de l'Ecole de Médecine, 75006 Paris, France.
Many experiments have demonstrated the human visual ability to perform some computation concerning a 3-D structure from the two-dimensional (2-D) motion of points or lines on a flat screen. Wallach and O'Connell (1953) studied this phenomenon, which they called the kinetic depth effect (KDE). Their stimuli were objects rotating between a light source and a translucent screen on which subjects viewed the shadowed objects.

In 1959, Green introduced computer generated stimuli making possible a systematic approach to the KDE. Given a set of points randomly positioned over a 3-D object, the loci of the projections of these points on a flat screen were calculated for various positions of the object in 3-D motion. With each position was thus associated a picture devoid of 3-D information. However, a viewer could perceive the 3-D structure of the object when the pictures were displayed successively at an optimal frequency.

This method has been extensively used for the study of 3-D perception from motion parallax (see e.g., Braunstein, 1962; Lappin, Doner, \& Kottas, 1980; Petersik, 1979) and, more recently, by Todd (1984) for the study of visual discrimination between cylindrical surfaces of different curvatures, with each cylinder rotating around its axis.

In addition to those psychophysical studies, and often independently of them, the field of computer vision has yielded a number of models for 3-D visual perception from moving images. Among these are algorithms based on optical flow differentiation, proposed by LonguetHiggins and Prazdny (1980), Waxman and Ullman (1985), and Subbarao (1986). Their approaches, as well as their limits of applicability and physiological plausibility, have been reviewed in a previous paper (Droulez 
\& Comilleau-Pérès, 1989). They show that 3-D characteristics (e.g., surface orientation) can be related directly to the first spatial derivatives of the optical flow. Using the same theoretical framework, we demonstrated that surface curvature is related to a second spatial derivative of the velocity field, the spin variation (SV). We proposed a neurophysiological scheme for SV processing by visual neurons. The goal of the present study was to assess the validity of predictions about the perception of curvature from motion, which are based on the SV model. Before describing the experiments, we outline the foundations of our theoretical approach.

\section{THEORETICAL BACKGROUND}

Our theoretical approach was based on the notion of SV. $\mathrm{SV}$ is a second spatial derivative of the image velocity field, which quantifies the bending of an image line during motion. It can be illustrated as follows. Let $S$ be a cylindrical surface, $K$ a point of $S$, and $O$ its retinal image (Figure 1). Initially, $S$ is supposed to be normal to axis $O K$ in point $K$, and circle $C$ of $S$ passing through $K$ projects as a straight line $C^{\prime}$ on the observer's retina. As $S$ is moving with a translation $T_{p}$ parallel to its axis (Figure 1A), $C^{\prime}$ turns into an arc of ellipse $C^{\prime \prime}$. This bending of image line $C^{\prime}$ is quantified by the value of SV in the horizontal direction. Alternatively, if $S$ is moving with a frontal translation $T_{0}$ orthogonal to its axis (Figure 1B) $C^{\prime}$ does not bend during the motion, and $S V$ is null along the horizontal direction.

To give the mathematical expression of SV, we use the following cartesian coordinate systems, illustrated in Figure 2: $O x_{0} y_{0}$ is a fixed coordinate system of the retina, $O x y$ is a coordinate system of the retina, which is rotated by an angle $\theta$ relative to $O x_{0} y_{0} ; E X Y Z$ is a coordinate system of the object space, where $E Z$ is the line of sight, and $E X$ and $E Y$ are frontal axes of retinal projections $O x$ and $O y$, respectively.

If $v$ is the $y$ coordinate of the retinal velocity field, the spin variation along axis $O x$ is a function of angle $\Theta$ given by

$$
\operatorname{SV}(\theta)=\frac{\partial^{2} v}{\partial x^{2}}
$$

If the 3-D movement of surface $S$ is decomposed into a rotation around the nodal point, $E$, of the eye, a frontal translation $T_{f}$, and a translation in depth, it can be shown (Droulez \& Cornilleau-Pérès, 1989) that SV depends only on the surface curvature and orientation and on $T_{f}$. If $Z(X, Y)$ is the equation of surface $S$, and if $T_{f Y}$ is the $Y$ component of $T_{f}, \operatorname{SV}(\theta)$ is related to the normal curvature of $S$ in the direction $\theta, c_{N}(\Theta)$, by the equation

$$
\begin{aligned}
\operatorname{SV}(\theta)= & -c_{N}(\theta) \cdot T_{f Y} \\
& \cdot\left(1+\frac{\partial Z^{2}}{\partial X}+\frac{\partial Z^{2}}{\partial Y}\right) \cdot\left(1+\frac{\partial Z^{2}}{\partial X}\right) .
\end{aligned}
$$

Let $X^{\prime}$ be the projection of axis $O x$ on the tangential plane to $S$ in $K\left(X^{\prime}\right.$ is parallel to $X$ in the particular case
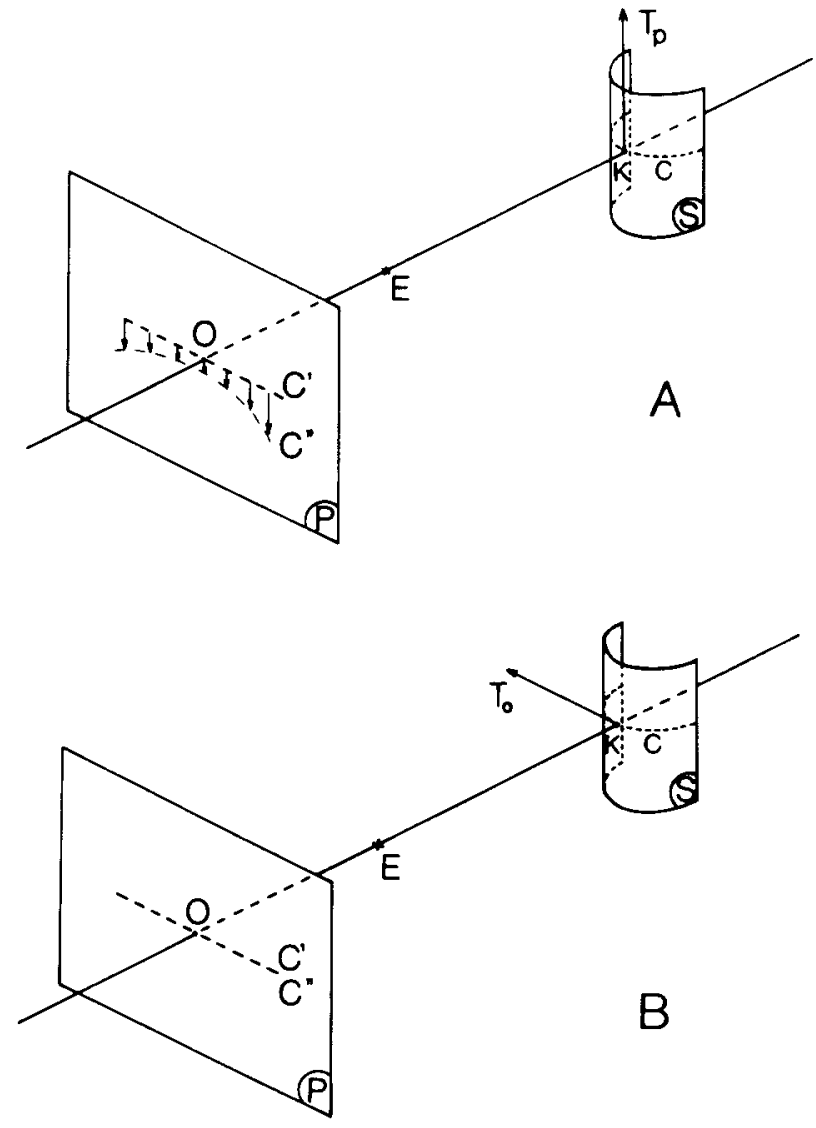

Figure 1. $S$ is a patch of cylinder moving in front of the eye, represented by its nodal point, $E$. $K$ is a point of $S$, and $O$ is the image of $K$ on the retina, which is here approximated by a plane. $S$ is assumed to be normal to line $E K$ before the motion. Initially, direction $C$ of maximum curvature of $S$ passing through $K$ (shown as a dotted line) projects onto the retina in a straight line, $C^{\prime}$. Panel $A$ : When $S$ is submitted to a frontal direction, $T_{p}$, parallel to the axis of $S, C^{\prime}$ turns into ellipse arc $C^{\prime \prime}$ during the motion. Panel B: On the opposite, when frontal translation $T_{0}$ is orthogonal to the axis of $S, C^{\prime}$ remains a straight line, $C^{\prime \prime}$, throughout the motion.

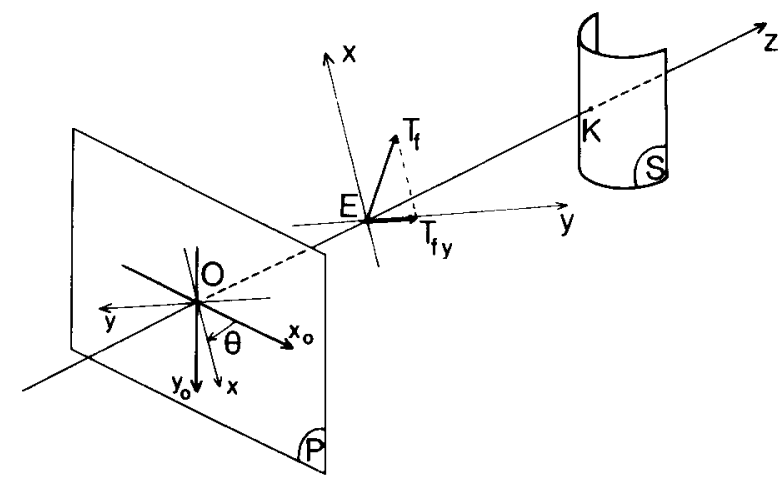

Figure 2. The different coordinate systems. $P$ is the plane of the retina, $O x_{a} y_{0}$ is a fixed coordinate system of $P$ from which the coordinate system $O x y$ is rotated by an angle, $\theta$. $E$ is the nodal point of the eye, and axis $E Z$ is normal to $P$. In the 3-D cartesian coordinate system EXYZ, axes $E X$ and $E Y$ project as $O x$ and $O y$ on $P$. $K$ is at the intersection of surface $S$ and axis $E Z ; S$ moves with frontal translation $T$, of $Y$-coordinate $T_{f}$. 
of Figure 2). $c_{N}(\theta)$ is defined as the inverse of the radius of the circle that is osculator, in $K$, to the arc located at the intersection of $S$ and the plane normal to $S$ and passing through $\boldsymbol{X}^{\prime}$.

From the above equation, it should be noted that, if $T_{f}$ is not null, SV is null in every direction if and only if the surface is locally planar. Therefore, the basic assumption of our study is that SV can be used as a visual cue for curvature detection by the visual system.

To test whether SV is null in a given image point, the visual system may use different types of SV measures (that are null if and only if SV is null in every direction), such as the mean SV absolute value, or the maximum of SV. To make quantitative predictions, we chose to use the following measure of SV:

$$
\mathrm{m}(\mathrm{SV})=\sqrt{\int_{0}^{2 \pi} \mathrm{SV}(\theta)^{2} d \theta}
$$

In the case of a cylindrical surface, the study of the SV function yields the following conclusions:

- $\mathrm{m}(\mathrm{SV})$ is proportional to the cylinder curvature (the inverse of its radius) and to the velocity of frontal translation.

- $\mathrm{m}(\mathrm{SV})$ is smaller if the frontal translation $T_{f}$ and the cylinder axis are orthogonal (case $\mathrm{O}$ ) than if they are parallel (case P). In Figure 1, these two cases are represented by $T_{f}$ lying along $T_{o}$ and $T_{p}$, respectively. As demonstrated by Droulez and Cornilleau-Pérès (1989), the ratio of the measures of SV in cases $P$ and $O$ does not depend on the cylinder radius and is equal to

$$
R_{O P}=\mathrm{m}(\mathrm{SV}, \mathrm{P}) / \mathrm{m}(\mathrm{SV}, \mathrm{O})=2.23 \text {. }
$$

Since no a priori knowledge of the object structure and motion is assumed, the SV measure must not depend on the choice of the coordinate axes. Other SV measures that verify this are the maximum of SV or its mean absolute value, for which the two properties above are still true, except that the values of $R_{O P}$ are 2.66 and 2 , respectively. Therefore, instead of using a precise value of $R_{o p}$, we shall consider that the magnitude of $S V$ is about twice as high in case $P$ as it is in case $O$.

These properties of SV lead to the following predictions: (1) The curvature corresponding to the threshold of discrimination between a cylinder and a plane should be higher by a factor of about 2 in case $O$ than in case $P$, and (2) sensitivity to cylinder curvature should increase with the translation velocity.

Predictions 1 and 2 can be tested by measuring the thresholds for the detection of the curvature of a cylindrical surface, using a forced-choice task with two alternatives, cylinder or plane. The different stimulus parameters are specified in the next section of the paper; the effects of two of them-the magnitude of the translation velocity and the direction of the cylinder axis relative to that of the translation-were investigated in particular, because they are related to the predictions.

\section{METHOD}

\section{Subjects}

The 8 subjects ( 4 women, 4 men) were 22-26 years old and had normal uncorrected vision. All of them were naive subjects and were paid volunteers.

\section{Apparatus}

All the stimuli were generated by an HP computer (A900) and presented on-line on an HP $1310 B$ display coated with a rapid-decay phosphor P31. The addressable area was $2048 \times 2048$ pixels, on a rectangle that was set to $29.26 \times 20.48 \mathrm{~cm}$.

The luminous sets of dots were displayed $72 \mathrm{~cm}$ in front of the subject, within a luminous fixed circle of diameter $8^{\circ}$ visual angle. The center of the circle was in the subject's sagittal plane, at eye level. Each dot subtended a visual angle of 1.34'. The actual resolution was $0.43 \mathrm{~mm}$. Because of the nonlinearity of the cathode tube and of the nonplanarity of its screen, the image geometry had to be corrected by polynomial adjustment of the image coordinates. An estimate of the residual errors due to image distortion and to image digitization leads to the conclusion that for the experimental conditions used in this study, if these errors were spread over the image as badly as possible, the same images could be obtained for cylinders of curvature smaller than $0.135 \mathrm{~m}^{-1}$ (distortion error) and $0.05 \mathrm{~m}^{-1}$ (digitization error). Thus, the inferior limit for the surface curvature that could be explored with our apparatus was about $0.1 \mathrm{~m}^{-1}$.

The luminance of the dots and of the circle was $0.9 \mathrm{~cd} / \mathrm{m}^{2}$, as measured with a Topcon Luminance Meter. Over 24 image sequences, the mean and standard error of dot number were 205 and 12 , respectively.

The subject's head was held still by a chinrest and a headrest and was surrounded by a black tunnel to prevent lateral vision. The experiments took place in a dark room. Except if otherwise specified, viewing was binocular.

\section{Design}

Six parameters could vary independently in our experiments: motion velocity, motion amplitude, motion direction, viewing duration, cylinder curvature, and cylinder orientation. The surface motion was periodic, and the period covaried with motion velocity and amplitude. Also, the number of cycles seen by the subjects covaried with this period and the viewing duration.

\section{Procedure}

Before starting the experiments, each subject served in a 1-h practice session, during which he/she was presented with image sequences representing planes or highly curved cylinders moving with large amplitude and velocity. In addition, the surface patches were small enough so that their apparent limits projected within the circular window. These training sequences yielded a straightforward perception of the underlying surfaces, which could be precisely described by the subjects. In the course of this practice session, the display parameters were gradually changed and finally reached the values actually used in the experimental sessions. While the subjects were getting familiar with the displays, they were never given any feedback but simply had to describe what they perceived from the displays. For curvatures ranging from 33.33 to $10 \mathrm{~m}^{-1}$, the subjects gave correct descriptions of the cylinder orientations and could discriminate them from the plane.

The subjects then completed several 2-h trial experiments. Their responses were taken into account only after they had stabilized $*$ In spite of this precaution, a slow drift in the subjects' responses over time was unavoidable, but it was counterbalanced by limiting the critical comparisons of the results to those obtained on the same day. 
Unless otherwise specified, the motion and display parameters and the cylinder radius were fixed for a given experimental session. Before the session started, the subject was shown a picture representing the arc of a circle matching the profile of the cylinder he/she was to discriminate from the plane. The subject could thus estimate the difficulty of the test. $\mathrm{He} / \mathrm{sh}$ was also told whether the cylinder axis was horizontal or vertical.

After $8 \mathrm{~min}$ of mesopic adaptation, the subject was presented with one block of 48 trials, consisting of the displaying of 24 moving cylinders and 24 moving planes in random order. Each trial started with the presentation of a $12-\mathrm{sec}$ image sequence. The subject then pressed a key corresponding to his/her response ("cylinder" or "plane"). No feedback was given. After a delay of $7 \mathrm{sec}$, the next sequence was displayed. Between trials a fixed image was displayed, so that the subject was exposed to a constant level of luminance throughout the session.

The sensitivity to surface curvature was measured by the percentage of correct responses (PCR) over a session of 48 trials. The curvature threshold was defined as the curvature eliciting a PCR of $75 \%$ (the $50 \%$ PCR corresponding to chance level).

\section{Stimuli Parameters}

Surface motion. As stated earlier, the motion of surface $S$ is decomposed into a rotation around nodal point $E$ of the eye, a frontoparallel (or frontal) translation $T_{f}$, and an in-depth translation. Since the only component that is relevant for the perception of curvature from motion is $T_{j}$, we considered the other components as neutral parameters, which served us only in choosing the resulting movement of the surface. Two constraints governed this choice. First, this movement had to be the same for the cylinders and the plane (so that the subjects could not substitute 3-D motion discrimination for surface discrimination). The second constraint was related to the assumption that the perception of curvature is mediated by SV processing (i.e., by comparing the $2-\mathrm{D}$ velocities at various loci of the image). In view of Nakayama's (1981) finding that sensitivity to differential motion decreases when the overall velocity increases, we decided to adjust the motion parameters so that the overall image velocity was minimal. Those two constraints led us to choose for our tests a rotation around an axis, $X$, medial in the image area and tangent to the cylinder or plane (see Figure 3). Con-

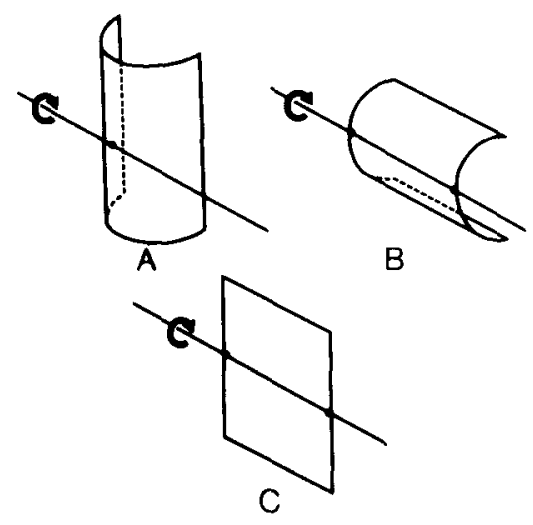

Figure 3. The surfaces displayed in the experiments and their motion. The subjects had to discriminate between a cylinder with either vertical axis $(A)$ or horizontal axis $(B)$ and plane $(C)$. The motion of all the surfaces was the same: a rotation around a horizontal line, in front of the subject, and tangent to the surface. Some of the surface points that remained fixed throughout the motion are indicated; they lie on the intersection between the surface and the axis of rotation.

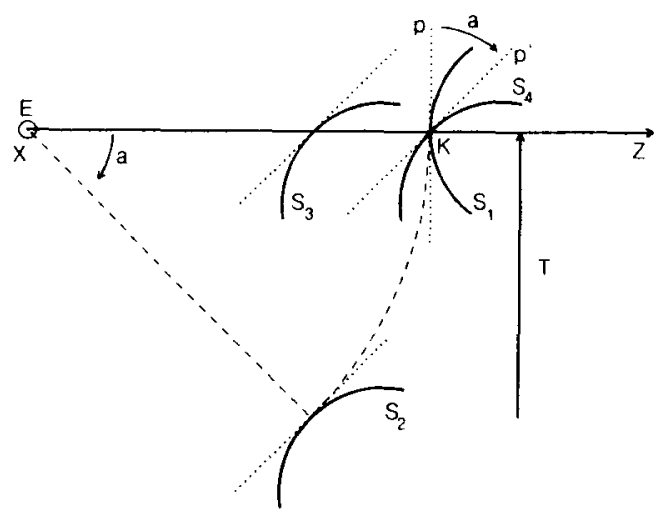

Figure 4. Decomposition of the surface motion. $E$ is the modal point of the eye, $(E Z)$ is the line of sight, and $T$ is the norm of the frontal translation that lies in the plane of the figure. The section of the surface by this plane is initially $S$ and intersects $(E Z)$ at point $K$. $(E X)$ is the axis normal to the figure plane. The surface is first rotated from position $S_{1}$ to position $S_{2}$ around $E X$, by angle $a$, which depends on $T$ and $E K$. It is then translated to the position $S_{3}$ through distance $T$, in a frontoparallel plane, and finally translated in depth to $S_{4}$ so that point $K$ is returned to its initial position. The resulting motion of the surface is a rotation around axis $(K X)$ of angle $a$. The plane tangent to the surface at $K$ is shown in dotted lines; it rotates from position $p$ to its final position, $p^{\prime}$.

sequently, the central point of the image (the projection of $K$ ) remained stationary for all the surfaces.

The decomposition of the resulting motion in a rotation around $E$ and a translation is described in Figure 4. Since point $K$ is fixed during the motion, the distance $D$ between points $K$ and $E$ is constant, and the motion amplitude may be characterized either by the rotation angle $a$, or by the norm $T$ of the frontal translation, which are related by the equation

$$
\sin a=T / D .
$$

Therefore, the motion of the surface relative to point $K$ was a pure rotation, while it presented a translation component in the viewer coordinate system centered in $E$. Actually, this motion was symmetrical relative to the median position $S_{1}$, and the surface oscillated sinusoidally around $K X$ between two extreme positions. Each image sequence depicted four oscillations of the surface for a total of 184 images displayed at a rate of $15.33 / \mathrm{sec}$.

Translation amplitude and velocity. The frontal translation is a key parameter of our experiments and was varied in velocity and amplitude. For the sake of comparison with results concerning the stereoscopic perception of 3-D objects, we used the interocular distance $\Delta=6.2 \mathrm{~cm}$ as the unity of the translation amplitude. Thus a frontal translation of total amplitude $T_{1}=5 \Delta$ corresponded to a translation of $15.5 \mathrm{~cm}$ in both directions from the central position, and to a total rotation of $S$ around $K X$ of angle $24.9^{\circ}$. As the oscillation period was $3 \mathrm{sec}$ (except if otherwise specified), this translation was completed with a 3-D velocity of mean $20.7 \mathrm{~cm} / \mathrm{sec}$ and maximum $31.6 \mathrm{~cm} / \mathrm{sec}$, which may be converted to an angular velocity of 3-D rotation around $K X$ of mean $16.6^{\circ} / \mathrm{sec}$ and maximum $26.03 \%$ sec.

The three parameters $-T$ (amplitude of frontal translation), $V$ (mean translation velocity over the image sequence), and $t$ (oscillation period)-are linked by the equation $2 \cdot T=V \cdot t$.

The values of those parameters were chosen under three constraints: (1) The mean velocity of the image points had to exceed the threshold for motion perception; (2) $T$ had to be commensurable with $\Delta$, the interocular distance, for future comparison with 
the perception of surface curvature in stereoscopic vision; (3) $t$ had to be higher than $1 \mathrm{sec}$. Our goal was not to study the perception of curvature in the limiting conditions of very brief motion. Rather, we intended to experiment under conditions of stable perception of surfaces. This is also why we usually presented several oscillations of the surface.

There exists a considerable amount of literature dealing with the lower thresholds of motion perception. A detailed review of this problem has no place here. The main result that is found is that, for a duration of presentation of more than $1 \mathrm{sec}$, the minimum perceived velocity is less than $1.5 \%$ sec (in some conditions, it may reach several seconds of arc per second) in foveal vision and increases in peripheral vision to a value of about $6^{\prime} / \mathrm{sec}$ at $8^{\circ}$ of eccentricity (Johnston \& Wright, 1985; Tynan \& Sekuler, 1982).

Finally, except in Experiment 3,t was set to a constant value of $3 \mathrm{sec}$, while $T$ had two possible values: $5 \Delta$ or $10 \Delta$. Under those conditions, the mean $2-D$ velocity of image points depicting a moving plane was $3.8^{\prime} / \mathrm{sec}$ (range $0-22^{\prime} / \mathrm{sec}$ ) for $T_{1}=5 \Delta$, and $13.7^{\prime} / \mathrm{sec}$ (range $0-57^{\prime} / \mathrm{sec}$ ) for $T_{2}=10 \Delta$. Although the 3-D motion velocity was twice as high for $T_{1}$ as for $T_{2}$, the image velocity does not follow the same rule, since the inclination of the object plane rela tive to the image plane increases nonlinearly with the motion amplitude. It also should be noted that the image velocity increased with the curvature of the cylinder.

A pilot experiment showed that the above motion parameters and an image rate of $15.33 / \mathrm{sec}$ elicited a compelling perception of a smooth oscillatory motion of the cylinders and planes. In all but one of our control experiments (Experiment 4), the absolute translation direction was vertical and, consequently, the rotation axis was horizontal.

Projection point and viewing distance. Retinal images are formed through the eye by an optical projection that may be approximated by a polar projection of center $E$, the nodal point of the eye. An observer thus views any object with a perspective defined by Braunstein (1962) as the ratio

$$
r=E F / E N,
$$

where $E N$ and $E F$ are the distances, along the line of sight, from $E$ to, respectively, the nearest $(N)$ and the farthest $(F)$ frontoparallel planes of the object. As we used a natural projection (i.e., the nodal point of the eye was the projection center) and a constant viewing distance $D$ from the screen, $r$ varied with the cylinder curva-

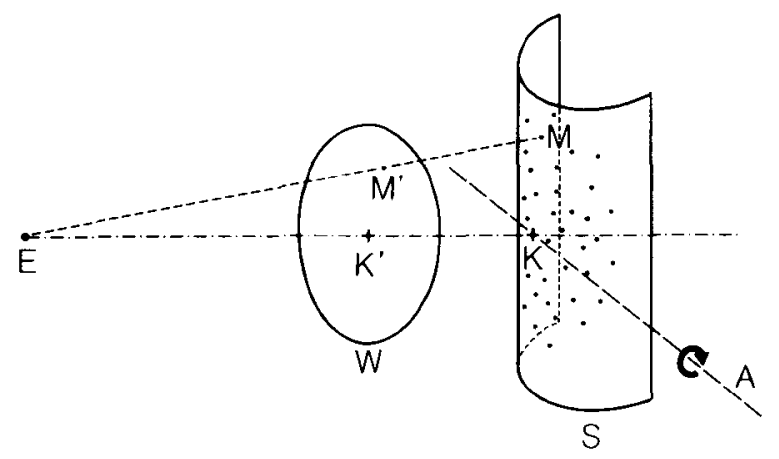

Figure 5. Image computation. Four-hundred dots were randomly spread over surface patch $S$ larger than that seen through circular window $W$. The projection of center $E$ of $\operatorname{dot} M$ was calculated and positioned at a point $M^{\prime \prime}$ of image. Point $K$ is at the intersection of the axis of rotation, $A$, with $S$, and projects on image point $K^{\prime} l o-$ cated in the center of $W$. Actually, points $K$ and $\boldsymbol{K}^{\prime}$ were superimposed in the experiments and were located in the plane of the screen. The calculation was repeated for different positions of $S$, depicting a rotation around $A$. ture and the position of the surface during the motion, between the values 1 and 1.13 .

Viewing area, dot number, and computation of the image. The flicker of the dots along the edges of the viewing area was avoided by setting a luminous border around this area. Braunstein and Andersen (1981) argued that "a rectangular border can serve as a cue to the flatness of a display, and is likely to reduce the realism of a simulated three-dimensional display." Accordingly, we displayed the border as a circle of diameter $8^{\circ}$ visual angle.

Petersik (1980) found that neither the number of dots nor their density significantly affected relative depth perception (5 to 60 dots spread within spheres of diameter $51^{\prime}$ or $4^{\circ}$ visual angle). However, Braunstein (1962) found that, as the number of dots located in a cube increased from two to six, the accuracy of relative depth perception increased, while the subjective coherence of the 3-D stimulus decreased. Unlike those previous studies, our experiments concern surfaces, rather than volumes, and thus presumably require a display of higher dot density (two dots in motion would not yield any perception of surface curvature). For instance, the cylinders used in Todd's (1984) study were defined by 100 dots. Moreover, we aimed to find curvature thresholds, using precise displays of the surfaces. For these reasens, we used images of about 205 dots.

Initially, for the median position of surface $S, 400$ dots were spread over a patch of $S$ larger than could be seen through the circular window in all the sequences (see Figure 5). Their 3-D coordinates were such that the density of their 2-D projections on the screen was uniform (this, of course, was true only for the median image of the sequence). The screen was tangent to the cylinder or plane in central point $K$ of the circular window (for the sake of clarity, this is not represented in Figure 5). Only image points that fell within the circular window were displayed.

\section{RESULTS}

\section{Experiment 1: Psychophysical Curves and Response Variability}

Six subjects took part in Experiment 1. The oscillation period was constant and equal to $3 \mathrm{sec}$; each trial consisted of the display of four oscillations of the surface, and the total viewing duration was therefore $12 \mathrm{sec}$. The amplitude of translation had two possible values, $5 \Delta$ and $10 \Delta$, the latter being therefore associated with twice the translation velocity of the former. The frontal translation was vertical; cases $P$ and $O$ thus refer to cylinders with vertical and horizontal axes, respectively. Each subject attended 12 sessions, for a given value of the motion amplitude, corresponding to each of six curvatures (between 0.5 and $10 \mathrm{~m}^{-1}$ ) in each of cases $P$ and $O$. Only 2 subjects, I.P. and F.N., performed the experiments involving both amplitude values $5 \Delta$ and $10 \Delta$. These 2 subjects also each completed five sessions associated with each of the curvatures 2.125 and $1 \mathrm{~m}^{-1}$. For each subject, the two sessions for a given curvature and motion amplitude, associated with cases $P$ and $O$, were run successively on the same day.

Figure 6 presents the mean data for 4 subjects and both motion amplitudes, and Figure 7 plots the individual performances of Subjects F.N. and I.P., for both motion amplitudes. For all experimental curves in Figures 6 and 7 , the PCR increased roughly linearly with the logarithm of the curvature for curvature values between 0.5 and 2 or $3 \mathrm{~m}^{-1}$; beyond this value, the performances stabilized at 


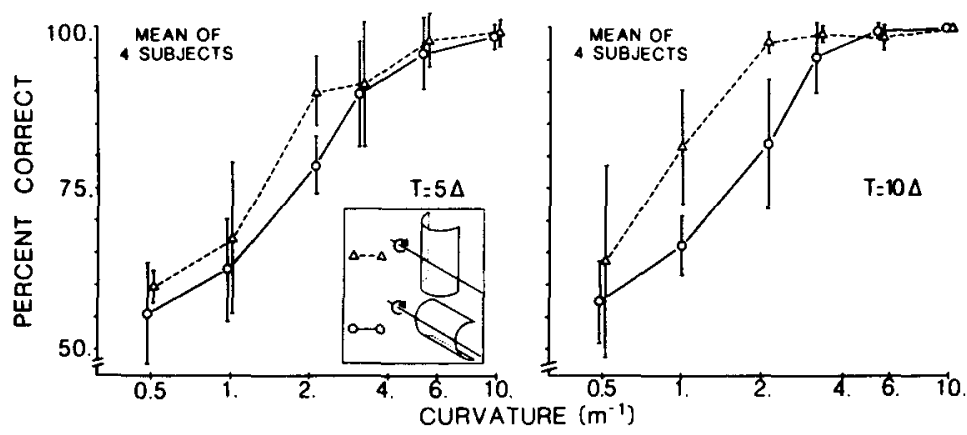

Figure 6. Psychophysical curves: mean for 4 subjects. Abscissae: curvature of the cylinder in $\mathrm{m}^{-1}$ on a logarithmic scale. Ordinates: percentage of correct responses in the discrimination between cylinder and plane. The frontal translation was vertical. Its amplitude was $5 \Delta$ (left) and $10 \Delta$ (right). Triangles, case $P$ (the cylinder axis was parallel to the frontal translation); circles, case $O$ (the cylinder axis was orthogonal to the frontal translation). Each point is the mean for four sessions, each completed by a different subject; a session consisted of 48 trials. The vertical bars represent the standard errors.

a good level of accuracy (the PCR was higher than 90\%). Both intersubject and individual variations (see standard errors plotted in brackets) are rather high: For all curvatures ranging from 0.5 to $2.125 \mathrm{~m}^{-1}$, the standard deviations of the PCR were between $1.6 \%$ and $15 \%$. In spite of these high variations, the subjects' performances in case $P$ were significantly better than they were in case $O$ at curvatures smaller than $3 \mathrm{~m}^{-1}$ (at higher curvatures, the PCRs were very close to $100 \%$ in both cases). Approximate curvature thresholds, corresponding to a PCR of
$75 \%$, may be linearly extrapolated from the curves of Figures 6 and 7. These thresholds are denoted $c_{P}$ and $c_{0}$ in cases $P$ and $O$, respectively, and reported in Table 1 for both motion amplitudes.

From Figures 6 and 7 and from Table 1, it clearly can be seen that Subject F.N. was more sensitive to surface curvature than was Subject I.P. The performances of these 2 subjects were, respectively, higher and lower than was the mean of 4 subjects (except for Subject I.P. and motion amplitude $10 \Delta$ ).
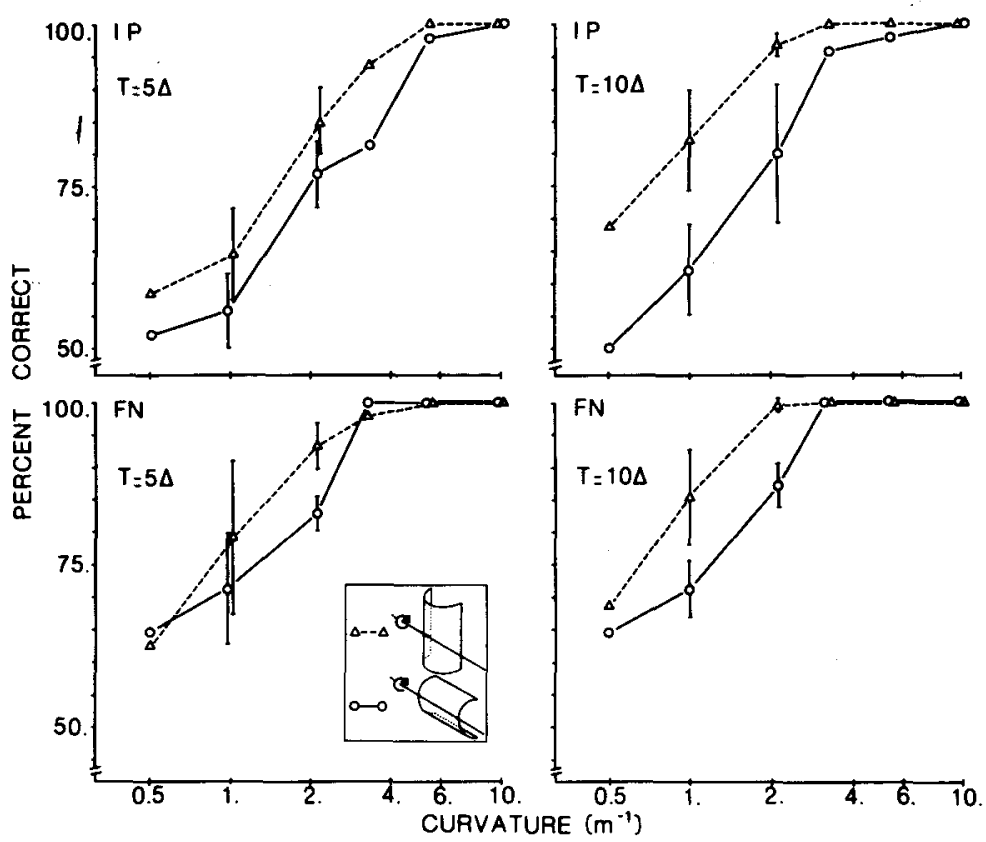

Figure 7. Psychophysical curves: individual performances of 2 subjects. Abscissae and ordinates, triangles and circles as in Figure 6. Subject I.P. (top), Subject F.N. (bottom). The motion amplitude $(T)$ was $5 \Delta$ (left) and $10 \Delta$ (right). Each point represents the result of one session (48 trials), except for the curvatures 1 and $2.125 \mathrm{~m}^{-1}$, where the data is the mean of five sessions. At these points, the standard errors are shown by the vertical bars. 
Table 1

Curvature Thresholds $\left(\mathrm{m}^{-1}\right)$ in Cases $P\left(c_{p}\right)$ and $O\left(c_{o}\right)$, and Their

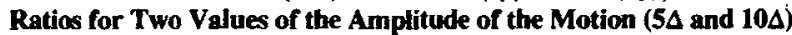

\begin{tabular}{ccccc}
\hline $\begin{array}{c}\text { Motion } \\
\text { Amplitude }\end{array}$ & Subject & $c_{P}$ & $c_{o}$ & $c_{o} / c_{P}$ \\
\hline $5 \Delta$ & All 4 & 1.30 & 1.80 & 1.38 \\
& I.P. & 0.87 & 1.24 & 1.43 \\
& F.N. & 1.47 & 1.97 & 1.34 \\
$10 \Delta$ & All 4 & 0.78 & 1.53 & 1.96 \\
& I.P. & 0.65 & 1.19 & 1.83 \\
& F.N. & 0.69 & 1.72 & 2.49 \\
\hline
\end{tabular}

The ratio $c_{o} / c_{P}$ (Table 1 , right column) matches quite well the predicted value of about 2, particularly for the large motion amplitude $(10 \Delta)$. This is confirmed by the curves of Figures 6 and 7, where the PCR is usually higher in case $P$ than in case $O$, this difference being significant for a motion amplitude of $10 \Delta$ and curvatures of 1 and $2.125 \mathrm{~m}^{-1}(p<.05)$. The difference was less marked for the motion amplitude $5 \Delta$ (it was significant only for a curvature of $2.125 \mathrm{~m}^{-1}$, with $p<.02$ ), and the ratio $c_{o} / c_{P}$ was only about 1.4 (Table 1 ), which is smaller than the predicted value.

Since the magnitude of SV is proportional to the translation velocity, the predicted ratio between curvature thresholds corresponding to $5 \Delta$ and $10 \Delta$ is 2 . In case $P$, this ratio was actually 1.67 (mean of data for 4 subjects), 1.34 (Subject I.P.), and 2.13 (Subject F.N.), whereas, in case $O$, it was only 1.18 (mean data of 4 subjects), 1.04 (Subject I.P.), and 1.15 (Subject F.N.). Therefore, the SV prediction concerning the influence of translation velocity on curvature perception is verified qualitatively, rather than quantitatively, to an extent that is greater in case $P$ than in case $O$. However, since both motion amplitude and velocity increased in Experiment 1, the difference between the predicted ratio and its experimental value might have been due to concurrent influences of those two parameters. This was questioned in Experiment 2.

\section{Experiment 2: Compared Influences of Oscillation Period and Motion Velocity and Amplitude}

In Experiment 1, the PCR was found to increase with simultaneous increases of translation amplitude $T$ and mean motion velocity $V$. Oscillation period $t$ was constant. Experiment 2 aimed at separating the influences of $T$ and $V$. Because the parameters $V, T$, and $t$ are linked by the equation $2 \cdot T=V \cdot t$, we had to vary two of them simultaneously, while the third was kept constant.

Subjects I.P. and F.N. attended eight successive sessions. The curvature was $1 \mathrm{~m}^{-1}$, and, for each of cases $P$ and $O$, the set of motion parameters was one of the following:

$$
\begin{aligned}
& \text { set 1: } T=5 \Delta ; V=V_{0} ; \quad t=t_{0} \\
& \text { set 2: } T=5 \Delta ; V=2 \cdot V_{0} ; t=t_{0} / 2 \\
& \text { set 3: } T=10 \Delta ; V=V_{0} ; \quad t=2 \cdot t_{0} \\
& \text { set 4: } T=10 \Delta ; V=2 \cdot V_{0} ; t=t_{0} \text {, }
\end{aligned}
$$

with $V_{0}=20.67 \mathrm{~cm} / \mathrm{sec}$, and $t_{0}=3 \mathrm{sec}$.
As the total duration of viewing was kept constant $(12 \mathrm{sec})$, the numbers of oscillations were $4,8,2$, and 4 for sets $1-4$, respectively. The data from both subjects are plotted in Figure 8, where each column corresponds to one constant parameter $-V, T$, or $t$.

The left column shows that, for a constant mean velocity of motion, the sensitivity to curvature was somewhat impaired by an increase of the translation amplitude. This effect was not systematic for Subject I.P., but the average PCR decreased for both subjects; this decrease was $5.55 \%$ for I.P. (discarding the curve located below the $50 \%$ level of chance) and $8.33 \%$ for F.N. Although these values are not significant, this tendency to decrease is not predicted by SV theory, because magnitude of SV slightly increases with the slant of the surface relative to the line of sight. For a given motion velocity, the average $S V$ increase is about $2 \%$ in case $P$ and $7 \%$ in case $O$ when motion amplitude increases from $5 \Delta$ to $10 \Delta$. These values are rather small, howęver, and we could not expect to find a significant increase of the PCR, considering the high response variability.

By contrast, motion velocity strongly modified the PCR (middle column of Figure 8 ) in the direction predicted by the SV theory; the systematic large improvement in sub-

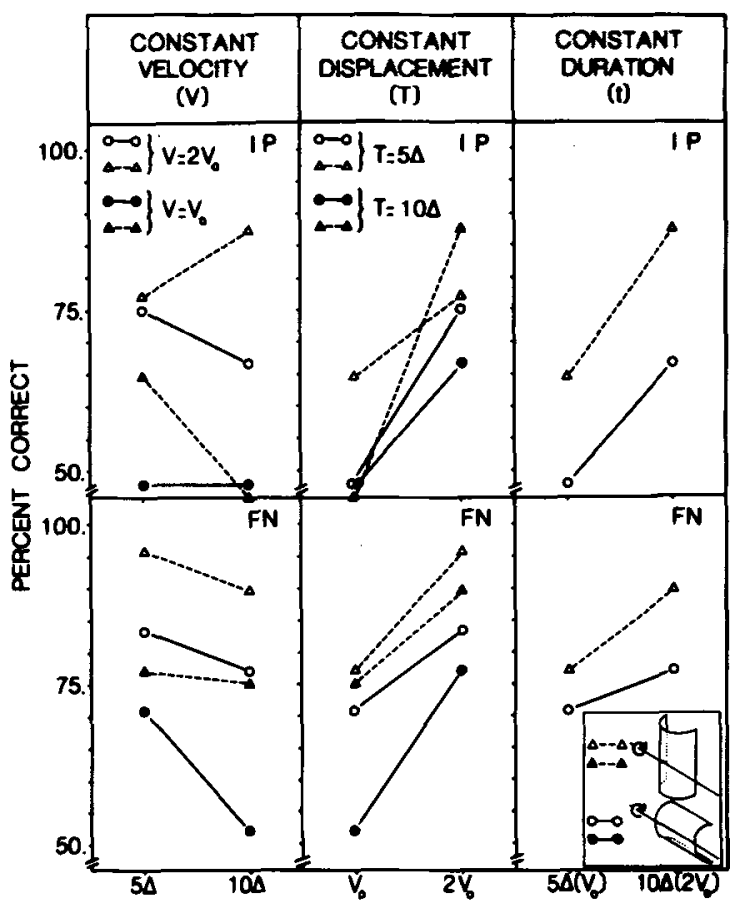

Figure 8. Compared infuences of velocity $(V)$, amplitude $(T)$ and duration $(t)$ of motion. The top and bottom rows plot the results from subjects I.P. and F.N., respectively, for curvature at $1 \mathrm{~m}^{-t}$. In each column are shown the curves corresponding to the constancy of the parameter indicated at the top. Abecisene: amplitude of trang lation (eft), velocity of translation (middle), and amplitude and velocity of translation (right). Ordinates: as in Figure 6. Triangles, case P; circles, case $O$. Filled and open symbols indicate different values of the velocity (left column) or the amplitude (middle column) of the motion. 
jects' performances (17.7\% for F.N. and $23.96 \%$ for I.P.) with 3-D motion velocity $V$ is in agreement with the linear increase of magnitude of SV with $V$.

The right column in Figure 8 illustrates the combined effect of amplitude and velocity of motion. As expected from the left and center columns, the impairment of sensitivity due to a $100 \%$ increase in motion amplitude was largely overwhelmed by the improvement due to a $100 \%$ increase in motion velocity. Consequently, the PCR increased with $T$ and $V$, as the duration of the motion (the oscillation period) was kept constant.

Therefore, among $V, T$, and $t$, and for the values of motion parameters used in Experiment 2, the critical parameter turned out to be motion velocity $V$; an increase of $V$ caused a larger improvement in the subjects' performances if the translation amplitude $T$ was kept constant than if the motion duration $t$ was kept constant. As in Experiment 1, for each set of parameters (represented in Figure 8 as either filled or open symbols), the PCR was higher in case $P$ (triangles) than in case $O$ (circles).

In Figure 9, some results of Experiment 1 have been replotted to allow a direct comparison with the right column of Figure 8. The results are from the same sub-

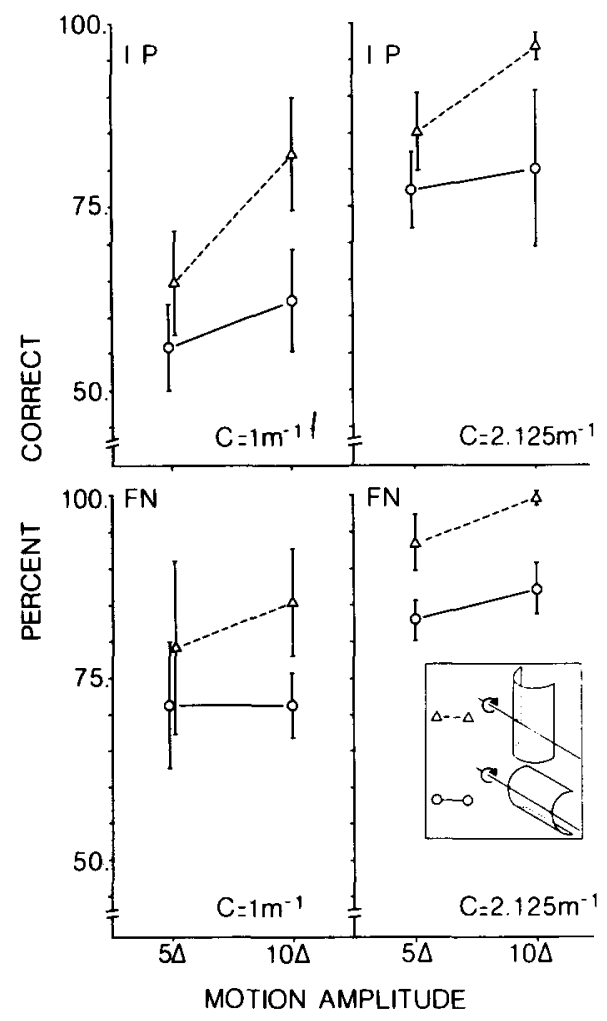

Figure 9. Influence of the amplitude of the motion. The top and bottom rows plot the results from Subjects I.P. and F.N., respectively, as obtained in Experiment 1 . The oscillation period was constant $\left(3\right.$ sec). The curvature values are $1 \mathrm{~m}^{-1}$ (left) and $2.125 \mathrm{~m}^{-1}$ (right). Abscissae: amplitude of translation. As this increases from $5 \Delta$ to $10 \Delta$ the 3-D motion velocity also doubles. Ordinates: as in Figure 6. Triangles, case $P$; circles, case 0 . The standard errors for five sessions are shown by vertical bars.

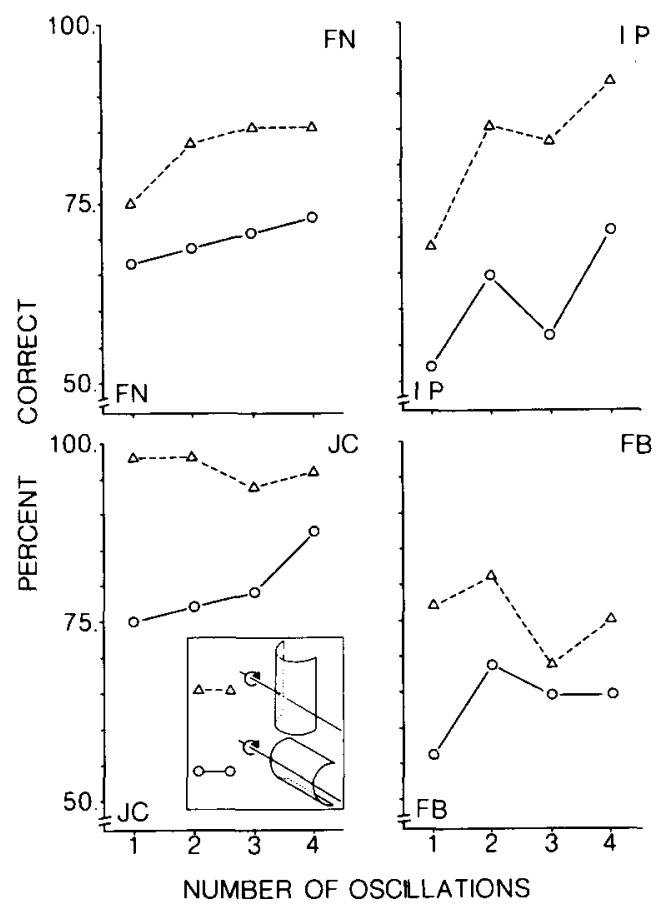

Figure 10. Influence of the duration of the viewing. Data from 4 subjects (F.N., I.P., J.C., F.B.). Abscissae: number of oscillations (the oscillation period was $3 \mathrm{sec}$ ). Ordinates: as in Figure 6. Triangles, case $P$ (cylinder axis parallel to the frontal translation); circles, case $\mathbf{O}$ (cylinder axis orthogonal to the frontal translation).

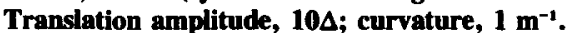

jects and support the previous conclusions all the more, since they hold for two curvatures and each point is the mean of five sessions. From Figures 8 and 9, it appears that the improvement due to increased velocity of the motion, in conjunction with an increase of its amplitude, is less marked in case $\mathbf{O}$ than in case P. Only in the latter case was it found to be significant for Subject I.P. and both curvatures $(p<.02)$ as well as for Subject F.N. and curvature $2.125 \mathrm{~m}^{-1}(p<.05)$.

Finally, these results might explain that the decrease of curvature threshold with motion velocity was smaller than had been predicted in Experiment 1 . As both velocity and motion amplitude increased, the performance remained poorer than if the amplitude had been kept constant.

\section{Experiment 3: Influence of the Duration of Viewing}

In the previous experiments, the subjects viewed sequences of four surface oscillations of $3 \mathrm{sec}$ each, for a total duration of $12 \mathrm{sec}$. In Experiment 3, our goals were to evaluate the role of this viewing duration and to verify that the anisotropy between cases $\mathrm{P}$ and $\mathrm{O}$ did not depend on this parameter. We examined the influence of a reduction of the total duration of viewing from 12 to $3 \mathrm{sec}$ by varying the number of oscillations of the surface in the range from 4 to 1 . The translation amplitude was constant $(10 \Delta)$, as were the oscillation period $(3 \mathrm{sec})$ and the cylinder curvature $\left(1 \mathrm{~m}^{-1}\right)$. Figure 10 presents the data 
Table 2

Means $(M)$ and Standard Errors (SE)

of the 2-D Velocity Magnitudes (in arcmin/sec) of Image Dots Randomly Located on Cylindrical Surfaces of Different Curvatures

\begin{tabular}{lccccc}
\hline & \multicolumn{2}{c}{ Case P } & & \multicolumn{2}{c}{ Case O } \\
\cline { 2 - 3 } \cline { 5 - 6 } Curvature $\left(\mathrm{m}^{-1}\right)$ & $M$ & $S E$ & & $S E$ \\
\hline 5.5 & 5.25 & \pm 0.16 & 4.93 & \pm 0.22 \\
3.25 & 4.49 & \pm 0.13 & 4.28 & \pm 0.19 \\
2.125 & 4.18 & \pm 0.14 & 4.09 & \pm 0.15 \\
0 (plane) & 3.85 & \pm 0.12 & 3.85 & \pm 0.12 \\
\hline
\end{tabular}

Note-The amplitude of the translation was $5 \Delta$.

from 4 subjects. The eight experimental points were obtained from eight consecutive sessions, conducted in random order all on the same day.

The PCR tended to increase with the duration of viewing, but the improvement was significant only when the number of oscillations increased from 1 to $4(p<.05)$. The difference between the PCR corresponding to cases $P$ and $O$ was roughly the same whatever the duration of viewing, a finding that supports the prediction of better performance in case $P$ than in case $O$.

\section{Experiment 4: Control Experiment:}

\section{About 2-D Velocity Magnitude as a Cue to Curvature}

The mean magnitude of image velocity cannot be used directly to recover surface curvature. However, since it was systematically higher for the cylinder than for the plane in a given session, it might have biased curvature judgments in the previous experiments. Therefore, we had to examine whether the subjects relied on this cue in judging the curvature.

Since each surface was defined by a set of randomly positioned points, the mean magnitude of the 2-D velocity over all the points throughout the motion varied from trial to trial for the same surface. In Table 2, the mean magnitude and its standard error (for 24 image sequences) are listed for four different curvatures, including the null curvature of the plane, and for a translation amplitude of $5 \Delta$. The mean 2-D magnitude of the velocity of points spread over a cylinder of curvature $c$ or over a plane, was found to be significantly different for planes and cylinders only when cylinder curvature was higher than $2 \mathrm{~m}^{-1}$. Therefore, we controlled the subjects' responses only for the three curvature values $5.5,3.25$, and $2.125 \mathrm{~m}^{-1}$.

The subjects were shown, in random order and always for the same viewing duration, 12 surfaces of each of the following types: (1) cylinder $C_{1}$ of curvature $c$ and translation amplitude $5 \Delta$, (2) cylinder $C_{2}$ of curvature $c$ and translation amplitude $(5-p) \Delta$, (3) plane $P_{1}$ of translation amplitude $5 \Delta$, and (4) plane $P_{2}$ of translation amplitude $(5-q) \Delta . p$ and $q$ were adjusted so that the mean 2-D velocity was the same for cylinder $C_{2}$ as for plane $P_{1}$ and the same for cylinder $C_{1}$ as for plane $P_{2}$. In a given session, the image sequences thus presented two values of the mean 2-D velocity, which, in contrast with the previous experiments, were not correlated with the type of surface. Parameters $p$ and $q$ increased with curvature $c$ of the cylinders and were always smaller than 1.1. The discrimination was more difficult for cylinder $C_{2}$ than for cylinder $C_{1}$ (as the former moved more slowly) but was easier for plane $\boldsymbol{P}_{2}$ than for plane $\boldsymbol{P}_{1}$; the global effect of the translation velocity on the subjects' performance was thus considered to be negligible.

If the subjects had relied on the magnitude of the velocity of the image in the sessions involving a constant amplitude of the motion, performance should have been worse for the sessions involving mixed amplitudes. Two subjects took part in Experiment 4; for each value of the curvature, the four sessions corresponding to cases $\mathrm{P}$ and $O$ with mixed or constant amplitude of motion were all held in succession on the same day. The results, presented in Figure 11, indicate no systematic relationship between the performance for mixed amplitudes and that for constant amplitudes of motion in either case $P$ or case $O$. Rather, the differences in performance seemed to fall well within the individual variability exhibited in Experiment 1 . Because performance was not particularly impaired, we conclude that curvature judgments were not based on discrimination between the average magnitudes of the 2-D velocity fields.

\section{Experiment 5: Influence of the Absolute Direction of Translation and of Binocular}

\section{Versus Monocular Viewing}

In Experiments 1-4, viewing was binocular and the translation was vertical (while the axis of rotation was horizontal). The first goal of Experiment 5 was to verify that the anisotropy observed in the subjects' performance

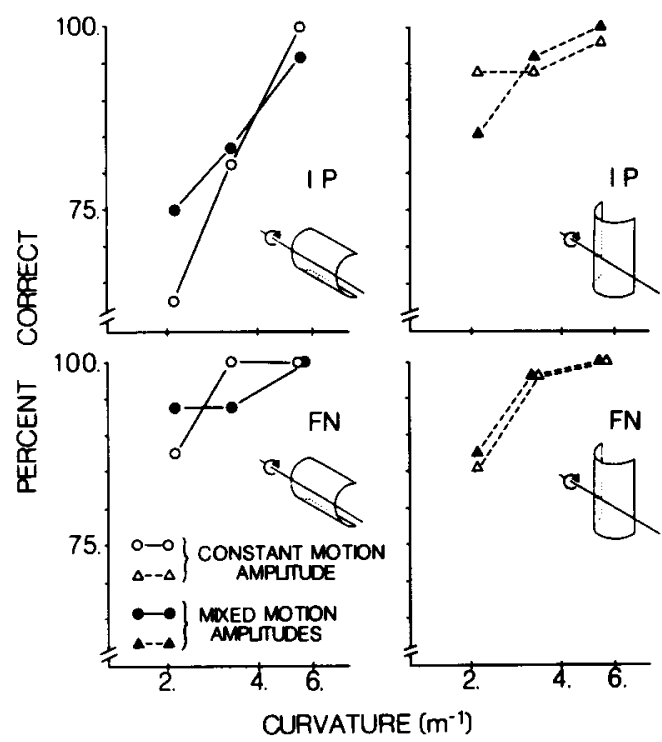

Figure 11. Control experiment: the average magnitude of the 2-D velocity field was not used as a cue for the detection of curvature. Results from 2 subjects (top row, I.P.; bottom row, F.N.) in cases $O$ (left, circles) and P (right, triangles). Abscissae and ordinates as in Figure 6. Open symbols are for sessions involving a constant amplitude of translation (5 5 ), and filled symbols are for sessions involving mixed amplitudes of motion (see text, Experiment 4). 
was not due to the absolute direction of the motion or cylinder axis but to the relative angle between those two directions. Therefore, here we compare the results obtained in cases $P$ and $O$ for vertical and horizontal translations.

Second, during binocular viewing of the stimuli, the binocular disparity of all image points is null and indicates that these points are spread over a planar surface (in the plane of the screen). By comparing monocular and binocular viewing conditions in Experiment 5, we intended to verify that the presence of a null disparity signal did not modify the subjects' responses or, at least, the relationship between responses in cases $P$ and $O$.

The 2 subjects who took part in Experiment 5 had not participated in previous sessions; hence, they were not used to any particular experimental conditions. Five times, each subject performed an experimental block consisting of eight sessions corresponding to the possible combinations of cases $\mathrm{P}$ and $\mathrm{O}$, monocular and binocular viewing, and vertical and horizontal translation. The curvature was set at $1 \mathrm{~m}^{-1}$, and the translation amplitude was set at $10 \Delta$. For monocular viewing, the subjects used the dominant eye (the right eye for Subject S.A., the left for Subject G.G.).

Figure 12 presents the results from both subjects for binocular viewing (left) and monocular viewing (right).

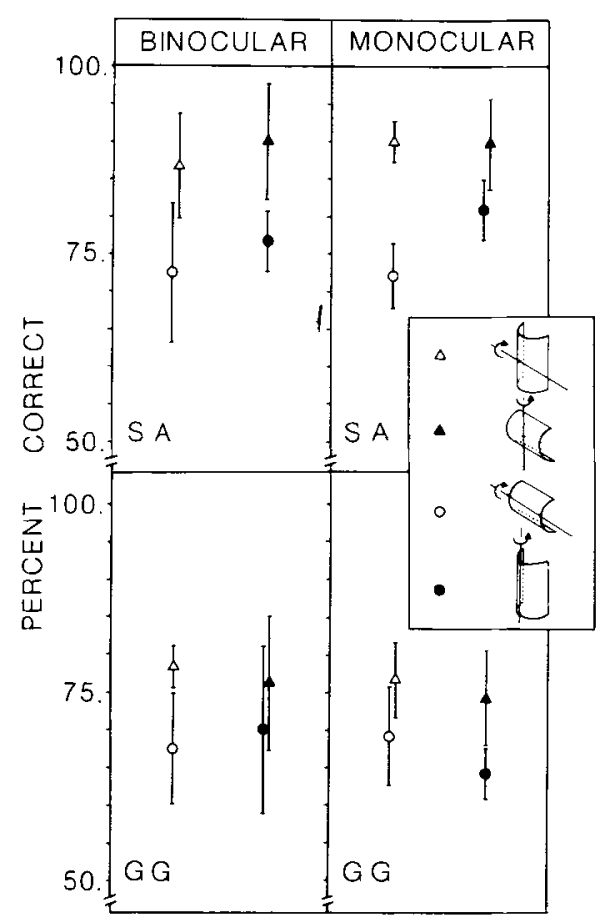

Figure 12. Control experiment: monocular versus binocular viewing, and absolute direction of translation. Each point corresponds to the mean of five sessions. The vertical bars show the standard errors. Triangles, case P; circles, case O. Top row, Subject S.A.; bottom row, Subject G.G. Left column, binocular viewing; right column, monocular viewing. Ordinates as in Figure 6. The frontoparallel translation was vertical (open symbols) or horizontal (filled symbols). The curvature was $1 \mathrm{~m}^{-1}$, and the translation amplitude was $10 \Delta$.
Within each graph in Figure 12, the comparison of triangles (case $P$ ) with corresponding circles (case $O$ ) indicates the influence of the absolute direction of the motion. Slight anisotropy is evident for both subjects, but with opposite effects; for most results, S.A. exhibited a better curvature sensitivity for the horizontal translation, whereas this preferential direction was vertical for G.G. whatever the direction of the cylinder axis was. However, this anisotropy was significant only for Subject S.A., viewing monocularly a cylinder in case $O$ (circles of top right graph).

Comparison between left and right columns of Figure 12 indicates no significant change for the subjects' performances in both cases $\mathrm{P}$ and $\mathrm{O}$ and for both translation directions. We conclude that binocular and monocular viewing were equivalent in our experiments. Subject S.A. exhibited systematically a better curvature sensitivity than did Subject G.G., this difference being significant $(p<.05)$ for four out of eight sets of parameters.

Finally, of most interest is the constancy of the relationship between performances in cases $P$ and $O$. For all experimental conditions, PCR was significantly higher in case $P$ than in case $O$.

\section{DISCUSSION}

Our results lead to the following conclusions:

1. Human observers can accurately discriminate between a cylinder and a plane describing the same 3-D motion, a rotation around a frontoparallel axis, even when these surfaces are only specified by a set of moving, uniformly spread dots, without apparent edges. Although the responses varied rather widely from subject to subject and from session to session for a given subject, the experimental data largely support the theoretical predictions based on the SV theory.

2. Cylinders in case $\mathbf{P}$ (axis parallel to the frontal translation) were better discriminated from the plane than were those in case $\mathrm{O}$ (axis orthogonal to the frontal translation). The difference in performance between cases $P$ and $O$ depended on the translation velocity and amplitude. For one of the two translation amplitudes we used, the ratio between the curvature thresholds in cases $P$ and $O$ was very close to the predicted value (about 2).

3. Similarly, the prediction of the critical influence of translation velocity was in agreement with the experimental results: an increase of this velocity from about $16^{\circ} / \mathrm{sec}$ equivalent frontal translation to $32 \% \mathrm{sec}$ strongly improved the subjects' performance.

4. In contrast, a pure increase of the frontal translation amplitude from $23.3^{\circ}$ to $40.73^{\circ}$ at constant velocity impaired the judgments of the curvature. This effect was not predicted by the SV theory but was found to be rather small and, generally, nonsignificant, when compared with the consequence of a velocity change.

5. Control experiments showed that the subjects did not rely on the average magnitude of the image velocity field to discriminate between a cylinder and a plane. We also verified that no significant differences were observed 
when the sequence was viewed with one eye rather than with two. Finally, a slight, but not systematic, anisotropy was observed when the absolute translation direction was changed from horizontal to vertical; this did not change the relationship between the performance in cases $P$ and $O$.

All control experiments confirmed that the two main parameters that influenced the subjects' sensitivity to curvature were the translation velocity and the angle between the translation direction and the cylinder axis.

It should be stressed that the application of the SV model to our experimental conditions involves an implicit assumption, namely, that the visual system is able to perform a velocity interpolation between the image points of our stimuli in order to process spatial derivatives of the velocity field. The output of this interpolation is likely to depend on the dot density and on the size of the stimulus. Therefore, the level of performance that is quantified by our data may be related to the value of those parameters.

Todd (1984) showed that subjects were able to discriminate accurately between cylinders of curvature 10.9, $10.2,8.9,6.7$, and $3.6 \mathrm{~m}^{-1}$, presented at a distance of $50 \mathrm{~cm}$ from the viewer. However, Todd's results are difficult to compare with ours for several reasons. First, his cylinders rotate around their axes, which implies that the subject might have discriminated the surfaces on the basis of their 3-D motions rather than curvatures. Second, the amount of frontal translation that is involved in the motion of each stimulus covaried with curvature between values of about $5 \Delta$ and $2 \Delta$, whereas this parameter was fixed in our experiments. Third, the simulated viewing distance also covaried with curvature in Todd's experiment, whereas it was fixed in ours. Finally, Todd used a viewing area of $19.8^{\circ} \times 26.6^{\circ}$, which is far larger than our $8^{\circ}$ stimulus diameter. In spite of all these differences, it should be pointed out that the range of curvature for which Todd obtained a good level of performance was located well above the value of $1.8 \mathrm{~m}^{-1}$, which yielded threshold performance in our experiments.

The visual sensitivity to differential motion was measured by Nakayama, Silverman, MacLeod, and Mulligan (1985), with patterns of random dots moving with a velocity of unique direction $D$. The movement amplitude varied as a sinusoidal function of the dot position along $D$ (compression waves) or along the direction orthogonal to $D$ (shear waves). For spatial frequency above 1 cycle per degree (cpd), the sensitivity to shear waves was found to be lower than for compression waves. Since our velocity fields were not unidirectional, they could not be approximated by pure compression or pure shear waves. Moreover, our stimuli presented low motion spatial frequencies (inferior to $0.1 / \mathrm{cpd}$ ), for which Nakayama et al. failed to observe any difference between the sensitivities to shear and compression motions.

Our results can also be compared with those obtained by Rogers and Graham $(1979,1982,1985)$ in a set of experiments on the sensitivity to visual depth evoked by motion parallax. Those authors instructed monocular ob- servers to move their heads from left to right while viewing a random dot pattern. Each head movement was correlated to a differential motion in the dot array, simulating the motion parallax field created by a 3-D corrugated surface. Thresholds for perceiving the simulated surface corrugations were measured by decreasing the simulated depth between peaks and troughs of the surface profile. In the study presented by Rogers and Graham (1985), the subject could move his/her head through $13 \mathrm{~cm}$, at $57 \mathrm{~cm}$ from the screen displaying the dot array. This corresponds to a frontal translation of about $2 \Delta$. The vertical profile of the simulated surface was a difference of Gaussians (DOG), whereas the horizontal profile was a straight line. This surface was thus locally equivalent to a horizontal cylinder, and the viewing conditions were comparable to case $P$ of our experiments.

For threshold comparisons, we liken a cylinder of curvature $c$, used in our procedure, to a DOG of angle $b=8^{\circ}$ between troughs, tangent to the cylinder in the center of the image and having the same extent in depth (i.e., the distances from the eye to the farthest points, located at $4^{\circ}$ eccentricity, are equal). $c$ is related to peakto-trough depth $d$ of the DOG and to viewing distance $D$, by the equation

$$
c=2 d /\left[D^{2} \cdot \operatorname{tg}^{2}(b / 2)+d^{2}\right] .
$$

In these conditions, Rogers and Graham (1985) found equivalent disparity thresholds of $18^{\prime \prime}$ and $35^{\prime \prime}$ for 2 subjects, corresponding to values of $d$ of 0.73 and $1.4 \mathrm{~mm}$ at a distance $D$ of $72 \mathrm{~cm}$ and to equivalent curvature thresholds of about 0.58 and $1.1 \mathrm{~m}^{-1}$, respectively. These values fall well within the range of curvature thresholds presented in Table 1, case P. However, more precise comparison is not easy because of the difference in the motion amplitudes used in the two experiments and the differences in curvature distributions between a DOG and a cylinder. Moreover, the total extent of the cylinder viewed in our experiments was $8^{\circ}$, whereas the DOG area viewed in Rogers and Graham's experiments presumably extended farther than the troughs. In addition, the motion velocity was not controlled in Rogers and Graham's experiments; the subject could move his head laterally at his own frequency. However, the typical frequency reported was $0.5 \mathrm{~Hz}$, which is comparable to the oscillation frequency of $0.33 \mathrm{~Hz}$ used in Experiment 1 of our study.

Also, of much interest is the anisotropy that Rogers and Graham described in the sensitivity to corrugated surfaces at low spatial frequencies (less than $0.1 \mathrm{cpd}$, a range that comprises our main spatial frequency of about $0.06 \mathrm{cpd}$ ). Rogers and Graham (1983) found that the "amount" of perceived depth is less when the depth corrugations are vertical than when they are horizontal (the head translation being horizontal). This result is in agreement with ours and is predicted by the SV theory. Yet it should be pointed out that Rogers and Graham used a matching task at supratheshold levels in conditions of self-motion rather than object motion. This suggests that our results might 
extend on one hand to suprathreshold curvatures (i.e., the perceived curvature would be larger for a cylinder $P$ than for a cylinder $\mathrm{O}$ ) and, on the other hand, to any motion of the observer relative to the surface. In addition, Rogers and Graham obtained the same directional anisotropy in the case of depth perception from binocular disparities. Indeed, the stereoscopic images of an object are obtained from each other by a translation along line $L$ joining both eyes. Therefore, it can be predicted that the thresholds for curvature detection in stereopsis will be lower if the cylinder axis and $L$ are parallel than they will be if they are orthogonal.

The results that we obtained are in agreement with our initial hypothesis, namely, that curvature detection is mediated by the detection of a second spatial derivative of the velocity field-the spin variation. However, one can wonder whether the anisotropy observed between cylinders $P$ and $O$ could be interpreted in terms of the first derivatives of the velocity field, and we have to check that those first derivatives cannot be used as cues in our discrimination task. Given a velocity field of coordinates $u$ and $v$ in a retinal orthogonal system of coordinates $(x y)$, we define the elongation and the angle variation in direction $x$ as derivatives $u_{x}$ and $v_{x}$ (see Note 1 for notational conventions), respectively, which are illustrated schematically in Figures 13A and 13B. Therefore, the elongation and angle variations along all directions can be quantified by the operators

$$
\begin{aligned}
& \text { ELONG }=\sqrt{\int_{0}^{2 \pi} u_{x}^{2} /(2 \pi) d \theta} \\
& \text { ANVAR }=\sqrt{\int_{0}^{2 \pi} v_{x}^{2} /(2 \pi) d \theta}
\end{aligned}
$$

where $\theta$ is the angle between axis $x$ and a fixed direction of the image. Combinations of the first derivatives of the velocity field also have been shown to be directly related to structure parameters of the surface. For instance, Koenderink (1985) demonstrated that a simple relationship exists between the 3-D orientation and motion of a surface and the following operators: the divergence of the velocity field, $u_{x}+v_{y}$, which characterizes a magnification (or minification) of the image (Figure 13C); and the deformation of the velocity field, $u_{x}-v_{y}$, which quantifies a dilatation in the $x$-direction, accompanied by a contraction in the $y$-direction (Figure 13D).

Contrary to the divergence, the deformation depends on the choice of axes $x$ and $y$. Therefore, we chose to quantify the local divergence and deformation of our images by the operators

$$
\begin{aligned}
& \mathrm{DIV}=\left|u_{x}+v_{y}\right| \\
& \mathrm{DEF}=\sqrt{\int_{0}^{2 \pi}\left(u_{x}-v_{y}\right)^{2} /(2 \pi) d \Theta} .
\end{aligned}
$$

The values of these four operators were calculated in every pixel of an artificial circular image of diameter 118 pixels; their mean and standard deviation over the image

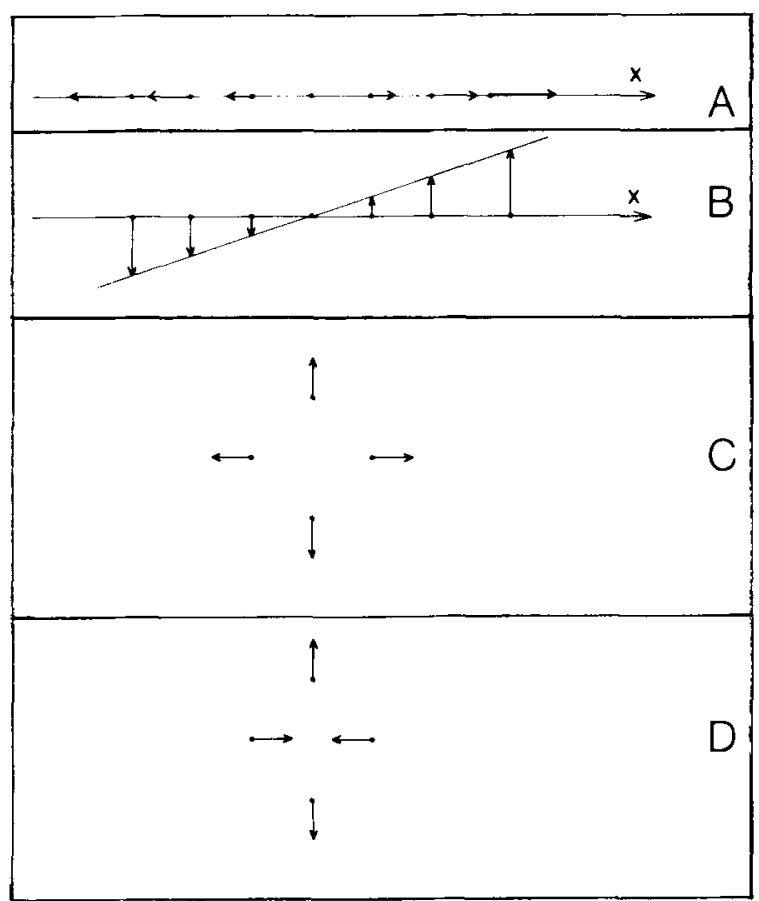

Figure 13. Schematic illustration of different types of image changes. Panel A: Elongation along the $x$-direction. Panel B: Angle variation along the $x$-direction. Panel C: Magnification (quantified by the divergence). Panel D: Deformation.

were also calculated. This was done for images representing cylinders of curvature $1 \mathrm{~m}^{-1}$ in cases $P$ and $O$ and in planes. We also considered two orientations of the surfaces: (1) normal to the visual axis (corresponding to the median image of our stimuli) or (2) inclined by an angle of $12^{\circ} 26^{\prime}$ or $25^{\circ} 30^{\prime}$ relative to this axis, corresponding to the extreme position reached when the motion amplitude is $5 \Delta$ or $10 \Delta$, respectively. All the parameters used for the computation were those of our experiments (in particular, the diameter of 118 pixels covered $8^{\circ}$ visual angle). This computation led to the following conclusions:

1. From one surface to another, each operator varied weakly, in mean value (less than $28 \%$ for the normal orientation, less than $3 \%$ for the two other orientations), and this variation was never statistically significant.

2. The mean and maximum values of each operator were always closer for cylinder $P$ and plane than for cylinder $O$ and plane.

Therefore, if based on any of the four first-order operators defined above, the cylinder-plane discrimination should be easier in case $O$ than in case $P$. This is in agreement with the fact that the first spatial derivatives of the velocity depend on the surface orientation, which differs more strongly, in every image point, between the cylinder and plane in case $O$ than in case $P$.

In view of the work presented by Nakayama et al. (1985), Rogers and Graham explain their results as the consequence of a different visual sensitivity to compression and shear transformation. We chose to interpret this 
mathematically in terms of the first spatial derivatives of the velocity field along itself (compression transformation), and along the direction orthogonal to it (shear transformation), by introducing the following operators in every image point $M$ of nonzero velocity:

$$
\begin{aligned}
\operatorname{COMP}(M) & =\left|u_{x}\right| \\
\operatorname{SHEAR}(M) & =\left|u_{y}\right| .
\end{aligned}
$$

Here $(x y)$ is an orthogonal coordinate system such that axis $x$ is colinear to the 2-D velocity. In the case of shear waves, COMP is null in every image point, whereas SHEAR is null for compression waves. COMP and SHEAR were also computed on the artificial images described above and again were found to be higher in case $O$ than in case $P$. Therefore, our results cannot be explained by a different visual sensitivity to the first spatial derivatives of the velocity along itself and along the direction orthogonal.

At this point of the discussion, it also should be mentioned that the first spatial derivatives of the optical flow depend only on the motion, depth, and orientation of the surface and cannot give any information about surface curvature. The control computations presented here also prove that the subjects did not use them as indirect cues in the discrimination task.

Conversely, is it possible to interpret Nakayama et al.'s results in terms of SV? A simple calculus indicates that measure $m$ of the SV is higher by a factor of 2.23 for shear waves than for compression waves. If related to the amount of SV, the detection of differential motion should be easier for shear waves than for compression waves, contrary to Nakayama et al.'s results. This discrepancy can be accounted for by the difference between the psychophysical task used by Nakayama et al. and that used by us. We had the subjects detecting curvature, which is directly related to SV, whereas Nakayama et al. studied the sensitivity to differential motion, which depends primarily on the first spatial derivatives of the velocity field.

Rogers and Graham interpret their data as the result of a different sensitivity to compression and shear transformations. Because they consider a pure frontal translation of the observer relative to the surface, their stimuli are effectively equivalent to pure shear or pure compression waves according to the orientation of the surface. In this respect, the fact that our surface movements were also composed of a rotation around the eye makes the whole difference: Contrary to Rogers and Graham's experiment, COMP and SHEAR are comparable for our cylinders $P$ and $O$. On the other hand, Rogers and Graham's stimuli present a strong SV anisotropy, just like in our experiment, because SV does not depend on the rotations around the eye. This illustrates the independence of the SV interpretation relative to the rotations around the eye, which is coherent with the theoretical independence of structure from motion perception relative to those rotations. We think that this is a strong argument in favor of an interpretation of Rogers and Graham's results in terms of SV rather than in terms of shear and compression.

Texture gives information about the 3-D structure of surfaces. Therefore, we also have to verify that our results cannot be explained by the changes in dot density. For the various surfaces and motions used in our experiments, the number and density of dots is the same for the median image of all of the sequences. However, the total number of dots displayed within the circular window is an increasing function of the slant of the surface relative to the visual axis. Therefore, the dot density on the image is smaller on the median image, where the surface is normal to the visual axis, than on those representing extreme positions of the surface during the motion. With the notational conventions used above, the increase of dot density (IDD) in the neighborhood of an image point is equal to the compression rate

$$
\text { IDD }=-\left(u_{x}+v_{y}\right),
$$

which is of the same sign over the whole image at a given instant. The results obtained for the operator DIV can thus be applied to IDD and show that the local dot density increases more with curvature for the cylinder $O$ than for the cylinder $P$. Since this property is verified in every image point, it is also true globally, and if the subjects relied on global or local changes in dot number, their performance should have been better in case $O$ than in case $P$.

From these control computations, we conclude that neither the first-order variations of the velocity field nor the changes in dot numerosity can account for the case $O$ case $P$ anisotropy.

$\mathrm{SV}$ is one of the second derivatives of the velocity field that is independent of the viewing distance (this can be easily verified in the SV calculus presented by Droulez $\&$ Cornilleau-Pérès, 1989). Therefore, the use of SV as a cue for surface curvature is in agreement with the results of Rogers (1986), who found that subject's ability to match surface curvature did not depend on the viewing distance. It also should be stressed that, because of its independence from the viewing distance, the SV scheme is valid for perspective projection, as well as for orthogonal projection, which is the limit of perspective when the viewing distance tends towards infinity.

More generally, the experimental data presented above supports the usefulness of a theoretical framework, such as SV theory. However, the existence of an SV computation in the visual pathway is far from being proved. Two paths for further investigation emerge: (1) psychophysical experiments have to be extended to different types of surface and to self-motion rather than to object motion, and (2) a search for SV detectors, such as those described by Droulez and Cornilleau-Pérès (1989), in the visual areas might provide the neurophysiological confirmation of SV theory. 


\section{REFERENCES}

Braunstein, M. L. (1962). Depth perception in rotating dot patterns: Effects of numerosity and perspective. Joumal of Experimental Psychology, 64, 415-420.

Braunstein, M. L., \& ANDersen, G. J. (1981). Velocity gradients and relative depth perception. Perception \& Psychophysics, 29, 145-155.

Droulez, J., \& Cornilleau, V. (1986). Adaptive changes in perceptual responses and visuomanual coordination during exposure to visual metrical distortion. Vision Research, 26, 1783-1792.

Droulez, J., \& Cornilleau-PÉrès, V. (1989). Visual perception of surface curvature: Spin variation theory and physiological implications. Manuscript submitted for publication.

Green, B. F. (1959). Kinetic depth effect (Psychology Group 58; Quarterly Progress Report). Cambridge, MA: MIT, Lincoln Laboratory.

JohnSTON, A., \& WRIGHT, M. J. (1985). Lower thresholds of motion for gratings as a function of eccentricity and contrast. Vision Research, $25,179-185$.

Koenderink, J. J. (1985). Space, form and optical deformations. In D. J. Ingle, M. Jeannerod, \& D. N. Lee (Eds.), Brain mechanisms and spatial vision (pp. 31-58). Dordrecht: Nijhoff.

LAPPiN, J. S., Doner, J. F., \& KotTAs, B. L. (1980). Minimal conditions for the visual detection of structure and motion in three dimensions. Science, 209, 717-719.

Longuet-Higgins, H. C., \& Prazdny, K. (1980). The interpretation of a moving retinal image. Proceedings of the Royal Society of London, B208, 385-397.

Nakayama, K. (1981). Differential motion hyperacuity under conditions of common image motion. Vision Research, 21, 1475-1482.

Nakayama, K., Silverman, G. H., Macleod, D. I. A., \&ulugan, J. (1985). Sensitivity to shearing and compressive motion in random dots. Perception, 14, 225-238.

Petersik, J. T. (1979). Three-dimensional object constancy: Coherence of a simulated rotating sphere in noise. Perception \& Psychophysics, 25, 328-335.

Petrersik, J. T. (1980). The effects of spatial and temporal factors on the perception of stroboscopic rotation simulations. Perception, 9 , 271-283.

Rogers, B. J. (1986). The perception of surface curvature from disparity and motion parallax cues. Investigative Ophtalmology \& Visual Science, Suppl. 27, p. 181.

Rogers, B., \& Graham, M. (1979). Motion parallax as an independent cue for depth perception. Perception, 8, 125-134.

Rogers, B., \& Graham, M. (1982). Similarities between motion parallax and stereopsis in human depth perception. Vision Research, 22, 261-270.

Rogers, B., \& Graham, M. (1983). Anisotropies in the perception of three-dimensional surfaces. Science, 221, 1409-1411.

Rogers, B., Graham, M. (1985). Motion parallax and the perception of three-dimensional surfaces. In D. J. Ingle, M. Jeannerod, \& D. N. Lee (Eds.), Brain mechanisms and spatial vision (pp. 95-111). Hingham, MA: Nifhoff.

Subbarao, M. (1986). Interpretation of image motion fields: Rigid curved surfaces in motion (Report No. CAR-TR-199). College Park: University of Maryland.

TODD, J. T. (1984). The perception of three-dimensional structure from rigid and nonrigid motion. Perception \& Psychophysics, 36, 97-103.

Tynan, P. D., \& Sexuler, R. (1982). Motion processing in peripheral vision: Reaction time and perceived velocity. Vision Research, 22, 1211-1217.

Wallach, H., \& O'ConNell, D. N. (1953). The kinetic depth effect. Journal of Experimental Psychology, 45, 205-217.

Waxman, A. M., Ullman, S. (1985). Surface structure and threedimensional motion from image flow kinematics. Intemational Journal of Robotics Research, 4, 72-94.

\section{NOTE}

1. The derivative of a function $u$ relative to a variable $x$ is written: $\partial u / \partial x=u_{x}$.

(Manuscript received October 10, 1988; revision accepted for publication March 3, 1989.) 